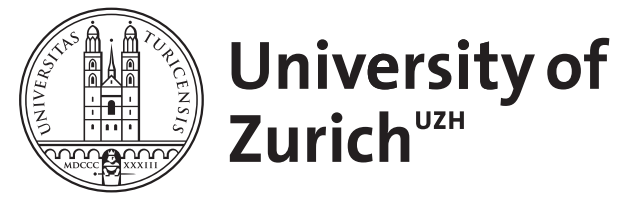

\title{
Ovarian toxicity from sirolimus
}

Braun, Matthias ; Young, James ; Reiner, Cäcilia S ; Poster, Diane ; Wüthrich, Rudolf P ; Serra, Andreas L

DOI: https://doi.org/10.1056/NEJMc1113145

Posted at the Zurich Open Repository and Archive, University of Zurich ZORA URL: https://doi.org/10.5167/uzh-66325

Journal Article

Originally published at:

Braun, Matthias; Young, James; Reiner, Cäcilia S; Poster, Diane; Wüthrich, Rudolf P; Serra, Andreas L (2012). Ovarian toxicity from sirolimus. New England Journal of Medicine, 366(11):1062-1064.

DOI: https://doi.org/10.1056/NEJMc1113145 
Maury J. Greenberg, M.D.

2500 Nesconset Hwy.

Stony Brook, NY

No potential conflict of interest relevant to this letter was reported.

THE AUTHOR REPLIES: Pollack's description of how his practice functions sounds like an example of the patient-centered medical home and how it should operate. My patient, Mary, would have benefitted from a more comprehensive approach to her care in which everyone involved was providing excellent care in his or her realm of expertise. I agree that working harder will not fix broken systems. Health care providers and their staff will need to go through major learning processes that will take money, time, hard work, and dedication to make this transformation a success. We desperately need the Obama administration to support and fund the efforts to provide this type of care. One lingering concern that needs to be addressed is the potential conflict of interest between physicians' payments and the care they provide to their patients. Even the perception that physicians have a financial disincentive to provide care will erode the primacy of the patient-doctor relationship.

Greenberg rightly points out that primary care is a community that includes not only internists but also family physicians and pediatricians. Each of these groups brings a different orientation to primary care, but all share a commitment to taking full responsibility for the "care" of the patient. It is a community of caregivers with whom I am proud to be identified. My story about Mary was not meant to be a complaint about my career or to reinforce inaccurate stereotypes. It was first and foremost a story about Mary, a courageous woman for whom I had the privilege to care. It was also an attempt to articulate the joys, sorrows, challenges, and ultimately the deep satisfaction that come from this type of work. My hope is that it might inspire and attract medical students to this immensely rewarding profession.

Diane R. Fingold, M.D.

Massachusetts General Hospital

Boston, MA

Since publication of her article, the author reports no further potential conflict of interest.

\section{Ovarian Toxicity from Sirolimus}

TO THE EDITOR: Sirolimus prevents rejection of renal allografts by blocking the mammalian target of rapamycin (mTOR), a signaling pathway known to regulate ovarian function. ${ }^{1}$ Sirolimus is being assessed as a treatment for autosomal dominant polycystic kidney disease (ADPKD). Observational data suggest a potential for ovarian toxicity, but this issue has not been evaluated in randomized, controlled trials. ${ }^{2}$

In the Suisse ADPKD study (ClinicalTrials.gov number, NCT00346918), a randomized, controlled trial conducted at the University Hospital Zurich from March 2006 through March 2010, we reviewed the occurrence of menstrual-cycle disturbances and the appearance of ovarian cysts ${ }^{3}$ in 39 women with ADPKD, an inherited kidney disease that is not known to affect ovarian morphology and function..$^{4,5}$ The trial was conducted in accordance with the principles of the Declaration of Helsinki, the Good Clinical Practice guidelines of the International Conference on Harmonization, and local regulatory require- ments. The local medical ethics committee approved the trial protocol. All patients provided written informed consent. A total of 21 women received sirolimus, and 18 received standard care for 18 months. Abdominal magnetic resonance imaging was performed in each patient every 6 months, and ovarian cysts larger than $2 \mathrm{~cm}$ in diameter were identified. Oligoamenorrhea was defined as no menstrual period for 3 months or more, or an interval of more than 35 days between menstrual periods. Analyses of prevalence were carried out with the use of exact logistic regression.

Wyeth (now Pfizer) provided the sirolimus but had no role in the trial design or in the collection, analysis, or interpretation of the data or the writing of the manuscript. The authors had full access to the study data and take responsibility for the integrity of the data, the accuracy of the data analysis, and the fidelity of this report to the trial protocol.

Among patients who received sirolimus at a 


\begin{tabular}{|c|c|c|}
\hline Variable & $\begin{array}{l}\text { Sirolimus } \\
(N=21)\end{array}$ & $\begin{array}{l}\text { Control } \\
(N=18)\end{array}$ \\
\hline \multicolumn{3}{|l|}{ Characteristic at randomization } \\
\hline Age $-y r$ & $31 \pm 8$ & $32 \pm 7$ \\
\hline Body-mass index† & $22 \pm 3$ & $22 \pm 3$ \\
\hline Estimated GFR $-\mathrm{ml} / \mathrm{min} / 1.73 \mathrm{~m}^{2} \leftarrow$ & $88 \pm 20$ & $96 \pm 16$ \\
\hline Menarche - yr $\int$ & $13 \pm 2$ & $13 \pm 2$ \\
\hline \multicolumn{3}{|l|}{ Parity - no. (\%)』 } \\
\hline Nulliparous & $13(62)$ & $14(78)$ \\
\hline$\geq 1$ child & $8(38)$ & $4(22)$ \\
\hline \multicolumn{3}{|l|}{ Contraception - no. (\%)』 } \\
\hline Hormonal & $10(48)$ & $9(50)$ \\
\hline Barrier method & $11(52)$ & $9(50)$ \\
\hline \multicolumn{3}{|l|}{ Oligoamenorrhea no./total no. (\%) } \\
\hline Enrollment & $3 / 21(14)$ & $2 / 17(12)$ \\
\hline Randomization & $2 / 20(10)$ & $1 / 15(7)$ \\
\hline $6 \mathrm{mo}$ & $9 / 21(43)$ & $2 / 18(11)$ \\
\hline $12 \mathrm{mo}$ & $5 / 20(25)$ & $2 / 18(11)$ \\
\hline $18 \mathrm{mo}$ & $5 / 19(26)$ & $1 / 18(6)$ \\
\hline Any visit after randomization & $11 / 21(52)$ & $3 / 18(17)$ \\
\hline \multicolumn{3}{|l|}{ Ovarian cysts — no./total no. (\%) } \\
\hline Enrollment & $4 / 21(19)$ & $4 / 16(25)$ \\
\hline Randomization & $2 / 21(10)$ & $4 / 17(24)$ \\
\hline $6 \mathrm{mo}$ & $6 / 21(29)$ & $2 / 14(14)$ \\
\hline $12 \mathrm{mo}$ & $6 / 20(30)$ & $3 / 17(18)$ \\
\hline $18 \mathrm{mo}$ & $7 / 18(39)$ & $2 / 17(12)$ \\
\hline Any visit after randomization & $12 / 21(57)$ & $5 / 18(28)$ \\
\hline
\end{tabular}

* Plus-minus values are means \pm SD. GFR denotes glomerular filtration rate.

$\uparrow$ The body-mass index is the weight in kilograms divided by the square of the height in meters.

The GFR was estimated with the use of the Chronic Kidney Disease Epidemiology Collaboration equation.

$\int$ Listed are data from patients' self-reported medical histories at randomization.

I Listed are patients' self-reported events during the 6 months before the visit.

dose of $1.5 \mathrm{mg}$ per day, 11 of 21 with ADPKD (52\%) reported menstrual-cycle abnormalities, as compared with 3 of 18 in the ADPKD control group (17\%) (Table 1) (odds ratio, 5.3; 95\% confidence interval [CI], 1.0 to 37.0). Five patients in the sirolimus group, as compared with only 1 patient in the control group, reported more than one episode of oligoamenorrhea. Ovarian cysts were detected in 12 of 21 patients in the sirolimus group (57\%), as compared with 5 of 18 in the control group (28\%) (odds ratio, 3.4; 95\% CI, 0.8 to 17.0) (Table 1). At more than one visit after randomization, ovarian cysts were detected in 6 patients in the sirolimus group, as compared with 2 in the control group. Differences in cycle disturbances were apparent in patients who were not receiving oral contraceptives; these differences were detected in 8 of 11 patients in the sirolimus group and in 2 of 9 in the control group. However, these differences were less apparent in patients receiving oral contraceptives ( 3 of 10 in the sirolimus group and 1 of 9 in the control group). Differences between the sirolimus and control groups with respect to the presence of ovarian cysts did not appear to depend on the contraceptive method. Ovarian cysts were detected in 7 of 11 patients who used barrier methods in the sirolimus group and in 3 of 9 who 
used barrier methods in the control group; they were detected in 5 of 10 patients who used oral contraceptives in the sirolimus group and in 2 of 9 who used oral contraceptives in the control group).

Low-dose oral sirolimus appears to increase the risk of menstrual-cycle disturbances and ovarian cysts. Monitoring of sirolimus-associated ovarian toxicity is warranted and might guide clinical practice with the use of mTOR inhibitors.

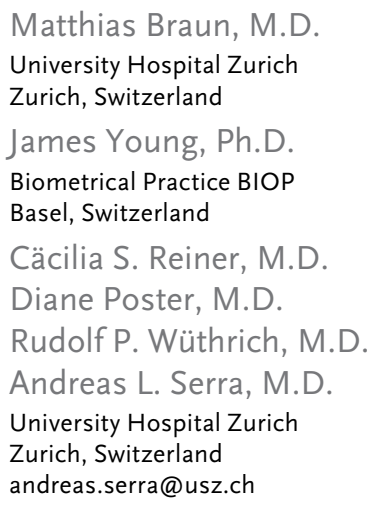

Supported by the Swiss National Science Foundation, the Polycystic Kidney Foundation, and an unrestricted research grant from Wyeth (now Pfizer). Wyeth Switzerland (now Pfizer) provided the sirolimus, and GE Healthcare Switzerland provided an Advantage Workstation for analysis of magnetic resonance imaging data.

Disclosure forms provided by the authors are available with the full text of this letter at NEJM.org.

1. Alam H, Maizels ET, Park Y, et al. Follicle-stimulating hormone activation of hypoxia-inducible factor- 1 by the phosphatidylinositol 3-kinase/AKT/Ras homolog enriched in brain (Rheb)/ mammalian target of rapamycin (mTOR) pathway is necessary for induction of select protein markers of follicular differentiation. J Biol Chem 2004;279:19431-40.

2. Cure P, Pileggi A, Froud T, et al. Alterations of the female reproductive system in recipients of islet grafts. Transplantation 2004;78:1576-81.

3. Serra AL, Poster D, Kistler AD, et al. Sirolimus and kidney growth in autosomal dominant polycystic kidney disease. $\mathrm{N}$ Engl J Med 2010;363:820-9.

4. Stamm ER, Townsend RR, Johnson AM, Garg K, MancoJohnson M, Gabow PA. Frequency of ovarian cysts in patients with autosomal dominant polycystic kidney disease. Am J Kidney Dis 1999;34:120-4.

5. Heinonen PK, Vuento M, Maunola M, Ala-Houhala I. Ovarian manifestations in women with autosomal dominant polycystic kidney disease. Am J Kidney Dis 2002;40:504-7.

\section{Pregnancy in a Woman with a Leptin-Receptor Mutation}

TO THE EDITOR: Leptin is considered to have an important role in reproductive functions, including menstrual-cycle regulation, pregnancy, and lactation..$^{1,2}$ The absence of leptin action caused by functional mutations in the leptin gene (LEP) or the leptin receptor gene (LEPR) has been linked to infertility in rodents and humans..$^{3-5}$ We report a pregnancy in a woman despite absent leptin signaling.

In 1998, we reported the case of a morbidly obese patient with a rare homozygous LEPR mutation, which was shared by several affected siblings. ${ }^{4}$ The mutation was found in the patient's blood and adipose tissue, indicating no evidence of chimerism. She had been followed for morbid obesity since early childhood, with abnormal compulsive-feeding behaviors and reduced levels of growth hormone and thyrotropin. ${ }^{4}$ She entered puberty late, with irregular cycles after the age of 17 years. Repeated evaluations of sex-hormone levels were considered to be normal after the age of 18 years. The patient underwent abdominoplasty at the age of 16 years and gastric-bypass surgery at the age of 24 years. Six months after gastric bypass, her weight had decreased from $220 \mathrm{~kg}$ (485 lb) to $170 \mathrm{~kg}$ (375 lb), with a concurrent decrease in the body-mass index (the weight in kilograms divided by the square of the height in meters) from 81 to 62 . She was counseled regarding contraception and was prescribed oral contraceptives. Two years after gastric bypass, just before an unplanned pregnancy, she had no diabetes, hypertension, respiratory disorders, or other recognized complications of obesity.

Ultrasonographic examinations during pregnancy were considered normal except for suspected macrosomia in the third trimester. The patient's total weight gain during pregnancy was $50 \mathrm{~kg}(110 \mathrm{lb})$ from a prepregnancy weight of $180 \mathrm{~kg}$ (397 lb). Routine screening for gestational diabetes was normal. Although occasional elevated blood sugar levels were documented during the pregnancy, the glycated hemoglobin level in the third trimester was 5.6\%. At 37 weeks 5 days of gestation (on the basis of firsttrimester ultrasonography), the patient delivered a son by elective cesarean section, which was performed because of breech presentation and suspected macrosomia under epidural anesthe- 